Research Paper

\title{
Protective Effects of Rosmarinic Acid against Selenite-Induced Cataract and Oxidative Damage in Rats
}

\author{
Chia-Fang Tsai ${ }^{1,2}$, Jia-Ying $\mathrm{Wu}^{2}$, Yu-Wen $\mathrm{Hsu}^{3}{ }^{凶}$ \\ 1. Department of Applied Cosmetology, National Tainan Junior College of Nursing, Tainan, Taiwan. \\ 2. Department of Biotechnology, TransWorld University, Yunlin County, Taiwan. \\ 3. Department of Optometry, Da-Yeh University, Changhua, Taiwan.
}

$\square$ Corresponding author: Hsu is to be contacted at the Department of Optometry, Da-Yeh University, No.168, University Rd., Dacun, Changhua 51591, Taiwan. Tel.: +886 4 8511888. E-mail address: yuwen@mail.dyu.edu.tw

(c) Ivyspring International Publisher. This is an open access article distributed under the terms of the Creative Commons Attribution (CC BY-NC) license (https:// creativecommons.org/licenses/by-nc/4.0/). See http://ivyspring.com/terms for full terms and conditions.

Received: 2018.12.12; Accepted: 2019.03.29; Published: 2019.05.10

\begin{abstract}
Cataracts are the major cause of blindness and are associated with oxidative damage of the lens. In the present study, the aim was to evaluate the protective effects of rosmarinic acid on selenite-induced cataractogenesis in Sprague-Dawley rat pups. The animals were randomly divided into five groups, each of which consisted of 10 rat pups. Group I served as normal control (vehicle administration). For testing cataract induction, animals of Groups II, III, IV, and V were administered a single subcutaneous injection of sodium selenite $(2.46 \mathrm{mg} / \mathrm{kg}$ body weight) on postpartum day 12 . After sodium selenite intoxication, Group II served as control selenite. From the 11 th day through the 17 th day, Groups III-V received rosmarinic acid intraperitoneally at doses of 5,10 , and $50 \mathrm{mg} / \mathrm{kg}$, respectively. On postpartum day 24 , the rat pups were examined for cataract formation, and the lenses were isolated for further analysis of proteins and oxidative damage indicators. Selenite caused significant $(p<0.05)$ cataract formation. Through the effects of selenite, the protein expressions of filensin and calpain 2 were reduced, and the calcium concentrations, the level of lipid peroxidation (TBARS), and inflammation indicators (iNOS, COX-2, and NFKB) were upregulated. Furthermore, the protein expression of the antioxidant status (Nrf2, SOD, HO-1, and NQO1), the antioxidant enzymes activities (GSH-Px, GSH-Rd, and catalase), and the GSH levels were downregulated. In contrast, treatment with rosmarinic acid could significantly $(p<0.05)$ ameliorate cataract formation and oxidative damage in the lens. Moreover, rosmarinic acid administration significantly increased the protein expressions of filensin, calpain 2, Nrf2, SOD, HO-1, and NQO1, the antioxidant enzymes activities, and the GSH level, in addition to reducing the calcium, lipid peroxidation, and inflammation indicators in the lens. Taken together, rosmarinic acid is a prospective anti-cataract agent that probably delays the onset and progression of cataracts induced by sodium selenite.
\end{abstract}

Key words: cataractogenesis, rosmarinic acid, sodium selenite, oxidative damage

\section{Introduction}

A cataract, defined as any opacity in the ocular lens caused by various etiological factors, is the major cause of blindness. When people suffer from cataracts, their vision and quality of life can be seriously impaired [1]. Though the standard treatment for cataracts is surgical intervention, which removes the opaque lens and replace it with an artificial intraocular lens, people cannot receive this procedure in many countries. Fortunately, recent studies have shown that experimental drugs designed to prevent degeneration of the lens can minimize the effects of cataracts.

The selenite cataract is a rapidly, clearly, and stably rodent model for the study of senile nuclear cataractogenesis, because experimentally seleniteinduced cataract response in animals is superficially 
similar to responses in human cataracts $[2,3]$. Thus, selenite-induced cataracts have been extensively used in experimental medical models to screen and evaluate the therapeutic potential of anti-cataract drugs. Even though the mechanism of cataract formation is not completely understood, the formation of senile cataracts is demonstrably associated with free radical-related oxidative stress [4]. Many studies have suggested that antioxidant supplements are successful in preventing oxidative stress-related cataract formation and oxidative damage [5, 6].

Rosmarinic acid (RA) is an ester of caffeic acid and 3,4-dihydroxy-phenyllactic acid [7], which has been found in more than 240 plant species [8]. Rosmarinic acid has several biological activities, including anti-inflammatory, anti-viral, anti-bacterial, anti-tumoral, and antiangiogenic properties. Many reports have indicated that rosmarinic acid serves as a photo-protective agent against UV exposure because of its inhibitory effects on skin photocarcinogenesis in vivo [9] and prevention of UVB-induced DNA damage in vitro [10]. Moreover, rosmarinic acid can inhibit cell proliferation and induce apoptosis of hepatic stellate cells [11]. Rosemarinic acid can also induce lymphoblastic leukemia cell death through a different cell death pathway [12]. However, only a small amount of evidence suggested that rosmarinic acid was effective in preventing ocular diseases. Recently, our group demonstrated that rosmarinic acid could inhibit the viability of human pterygium epithelial cells through the regulation of redox imbalance and induction of extrinsic and intrinsic apoptosis pathways [13]. Recent publications have shown that rosmarinic acid as a promising potential for treatment of cataract are reflected by its ex vivo and in vivo anti-cataract effects [14-17]. Unfortunately, these studies only reported that rosmarinic acid has the effect of inhibiting cataract formation, but did not elucidate the molecular mechanism by which rosmarinic acid inhibits cataract formation in vivo.

Because of the excellent bioactivity of rosmarinic acid, we hypothesized that supplementation with rosmarinic acid may protect against sodium selenite-induced cataracts in rats. Therefore, the aims of the present study were not only to investigate the protective effects of rosmarinic acid on sodium selenite-induced cataractogenesis in Sprague-Dawley rat pups, but also to further elucidate the anti-cataract molecular mechanisms of rosmarinic acid in enhancing the antioxidant defense system and inhibiting inflammatory in vivo. The extent of selenite-induced cataracts was also analyzed through histopathological observations.

\section{Methods}

\section{Animals}

Eleven-day-old Sprague-Dawley rat pups were obtained from the Animal Department of BioLASCO Taiwan Co., Ltd. (Taipei City, Taiwan). In each cage, ten pups and their mother were housed under normal laboratory environments. The animal room's relative humidity was maintained at $55 \pm 5 \%$ with a temperature of $25 \pm 2{ }^{\circ} \mathrm{C}$. All processes were completed according to the ARVO Statement for the Use of Animals in Ophthalmic and Vision Research.

\section{Treatment}

The animals were randomly divided into five groups, each consisting of 10 rat pups. Group I served as the normal control. To induce cataracts in the lenses, we gave the rat pups in Groups II, III, IV, and $\mathrm{V}$ a single subcutaneous injection of sodium selenite (2.46 mg/kg body weight) on postpartum day 12 . After sodium selenite intoxication, Group II served as sodium selenite (SE) control. In addition, Groups III, $\mathrm{IV}$, and $\mathrm{V}$ received rosmarinic acid intraperitoneally at doses of 5,10 , and $50 \mathrm{mg} / \mathrm{kg}$ body weight, respectively, from the 11th day through the 17th day. On day 12, the rat pups in Groups III, IV, and V received rosmarinic acid $1 \mathrm{~h}$ prior to sodium selenite injection. On postpartum day 24 , the rat pups were anesthetized with chloral hydrate and examined for cataract formation. After an assessment of the cataract formation, all animals euthanized and placed in a $\mathrm{CO}_{2}$ box. Lens samples were isolated and stored at $-70^{\circ} \mathrm{C}$ for further analysis.

\section{Evaluation of cataract formation}

At the final examination, the pupils were dilated with tropicamide $0.5 \%$ and phenylephrine hydrochloride $2.5 \%$. Each stage was graded and identified with the help of an expert ophthalmologist. Classification of the cataract stages was based on a scale of 0 through 6 [18]. Grade 0 was a normal clear lens; Grade 1 meant an initial sign of posterior subcapsular or nuclear opacity involving tiny scatters; Grade 2 indicated a slight nuclear opacity with swollen fibers or scattered foci in the posterior subcapsular; Grade 3 was a diffuse nuclear opacity with cortical scattering; Grade 4 meant a partial nuclear opacity; Grade 5 meant a nuclear opacity not involving the lens cortex; Grade 6 was a mature dense opacity involving the entire lens. The final numerical score was calculated by dividing the sum of each grade's number of affected rat pups by the total number of examined rat pups. Two observers without prior knowledge of the exposure and study groups performed all scorings. 


\section{Estimation calcium level in lens}

The dry weight of the lens was measured after heating at $100{ }^{\circ} \mathrm{C}$ for $12 \mathrm{~h}$. The lenses were then digested with $0.2 \mathrm{ml}$ concentrated $\mathrm{HCl}$ at room temperature overnight and adjusted to $1.0 \mathrm{ml}$ with deionized water. The mixtures were centrifuged at $10,000 \mathrm{~g}$ for $12 \mathrm{~min}$ to remove insoluble material. The calcium concentrations in the supernatant fractions were then measured by an atomic absorption spectrophotometer (model Spectra AA-3100, Perkin Elmer), operated with a slit width of $0.5 \mathrm{~nm}$, with the wavelength set at $422.7 \mathrm{~nm}$. Standard solutions were prepared from $\mathrm{CaCO}_{3}$ and deionized water. The results were expressed as mmol of calcium/gm dry weight of the lens.

\section{Measurement of catalase, GSH-Px and GSH-Rd activities, and GSH in lenses}

The homogenization procedure was performed under standardized conditions. Lens homogenates were prepared in cold Tris- $\mathrm{HCl}(5 \mathrm{mmol} / \mathrm{L}$, containing $2 \mathrm{mmol} / \mathrm{L}$ ethylenediaminetetraacetic acid, $\mathrm{pH}$ 7.4) using a homogenizer with a rotatory speed of 1,500 piston/min; three shifts downwards and upwards were performed. The unbroken cells and cell debris were removed by centrifugation at 10,000 $\times g$ for $10 \mathrm{~min}$ at $4^{\circ} \mathrm{C}$. The supernatant was used immediately for the catalase, glutathione peroxidase (GSH-Px), glutathione reductase (GSH-Rd), and GSH assays. The activities of these enzymes and GSH concentrations were determined according to the Randox Laboratories Ltd. kit instructions [13].

\section{Measurement of lipid peroxidation}

The quantitative measurement of lipid peroxidation was performed by measuring the concentration of thiobarbituric acid-reactive substances (TBARS) in the lens according to the method reported by Hsu et al. (2009). The amount of TBARS formed was quantified by the substances' reaction with thiobarbituric acid (TBA) and used as an index of lipid peroxidation. In brief, samples were mixed with a TBA reagent consisting of $0.375 \%$ TBA and $15 \%$ trichloroacetic acid in $0.25 \mathrm{M}$ hydrochloric acid. The reaction mixtures were placed in a boiling water bath for $30 \mathrm{~min}$ and centrifuged at $1811 \times g$ for $5 \mathrm{~min}$. The supernatant was collected, and its absorbance read at $532 \mathrm{~nm}$ with an enzyme-linked immunosorbent assay plate reader (Quant, BioTek, Winooski, Vermont, USA). The results were expressed as $\mathrm{nmol} / \mu \mathrm{g}$ of protein using the molar extinction coefficient of the chromophore $\left(1.56 \times 10^{-5} \mathrm{M}^{-1} \mathrm{~cm}^{-1}\right)$.

\section{Western blot analysis}

The protein concentrations of the lens homogenates were determined by the Bradford protein assay. The lens homogenates were separated by $10 \%$ polyacrylamide gel and transferred onto polyvinylidene fluoride membranes. After we incubated the membrane with blocking buffer $(5 \%$ nonfat milk in phosphate-buffered saline with Tween buffer) for $1 \mathrm{~h}$ at $4{ }^{\circ} \mathrm{C}$, the membranes were incubated overnight with specific primary antibodies in Trisbuffered saline (TBS) containing $0.1 \%$ Tween 20 at 4 ${ }^{\circ} \mathrm{C}$. The primary antibody was removed by washing the membranes 3 times in the TBS-T buffer and incubated for $2 \mathrm{~h}$ with the corresponding horseradish peroxidase conjugated secondary antibodies (1:2500) at $25^{\circ} \mathrm{C}$. After we washed the membranes three times in TBS-T, we developed the membranes using ECL Plus (GE Healthcare) and imaged them using an LAS-3000 Imaging System (Fujifilm).

\section{Histopathological evaluation}

After the animals were sacrificed with $\mathrm{CO}_{2}$, the eyes were removed, weighed, and fixed in Davidson's fixative. The eyes were processed for paraffin embedding following the standard microtechnique. Four- to five-micron sections of the eyes, stained with hematoxylin and eosin to estimate the lens damage, were observed under a microscope (IX71S8F-2, Olympus, Tokyo, Japan).

\section{Statistical analysis}

All values are expressed as the mean \pm SD. Comparison between any two groups was performed using a Chi-square or one-way analysis of variance (ANOVA) followed by Dunnett multiple comparison tests that used the statistical software SPSS (DR Marketing Co., Ltd. New Taipei City, Taiwan). A $p$ value $<0.05$ was considered statistically significant.

\section{Results}

\section{Morphological assessment of cataract formation}

Morphological examination of each rat pup's eyes provided important evidence of the cataract formation caused by sodium selenite. The lenses in all the animals in the normal control group were clear (Figure 1A). All the rat pups treated with the selenite alone developed moderate to severe cataracts (Figure 1B) that were graded as falling between stage 4 and stage 6 , indicating that our study had successfully established the selenite-induced cataract model. In contrast, we observed significantly greater amelioration in the extent of lens opacification in the groups treated with respective doses of 5,10 , and $50 \mathrm{mg} / \mathrm{kg}$ rosmarinic acid (Figures 1C, 1D, and 1E), than we did in those in the selenite-treated group. In group III, rat pups treated with $5 \mathrm{mg} / \mathrm{kg}$ rosmarinic acid had mild 
to moderate cataracts that were graded as falling between stage 2 and stage 4 , and $60 \%$ of the rat pups treated with $10 \mathrm{mg} / \mathrm{kg}$ rosmarinic acid had trace to mild cataracts that were graded as falling between stage 1 and stage 3 . Eight out of 10 rat pups in the group treated with a dose of $50 \mathrm{mg} / \mathrm{kg}$ rosmarinic acid had clear lenses. These morphological findings indicated that the cataract formation in the lens was effectively ameliorated when treated with rosmarinic acid.

Lens morphological examinations for cataract formation were recorded and scored, as shown in Figure 1F. In this semi-quantitative assessment, all scores of lens morphological examination in the selenite-treated group were significantly higher than those of the normal control $(p<0.05)$, indicating that the selenite had induced severe cataracts. All the tested doses of rosmarinic acid decreased the scores of cataract formation much more significantly $(p<0.05)$ than did the selenite-treated group, indicating that rosmarinic acid ameliorated selenite-induced cataract formation.

\section{Determination of calcium concentration in the lenses}

The mean calcium concentrations in the lenses of the five groups of rats on the 24th postpartum day are shown in Fig. 2A. The mean calcium concentration $(3.32 \pm 0.08 \mathrm{mmol})$ was significantly $(p<0.05)$ higher in the lenses of selenite-treated group than that in the lenses of all of the doses of rosmarinic acid treatment groups and that in normal controls $(0.75 \pm 0.07 \mathrm{mmol})$.

\section{Effects of rosmarinic acid on calpain 2 expressions in lenses}

Elevated calcium levels in selenite-treated animals prompted the comparison of calpain 2 activation. The results of the expressions of calpain 2 in the lenses are shown in Figure 2B. The calpain 2 expressions in the selenite-treated group were significantly lower than in the normal controls. Consistent with the lens morphological examinations, administration of rosmarinic acid significantly maintained the level of calpain 2 in the lens without being affected by selenite. The calpain 2 expressions in the rosmarinic acid-treated group were significantly higher than that in the selenite-treated control group $(\mathrm{p}<0.05)$. These findings indicated that the lenticular opacity being developed in the lenses were effectively inhibited by rosmarinic acid.

\section{Effects of rosmarinic acid on filensin expressions in lenses}

We used a western blot analysis to investigate the expressions of filensin in selenite-induced cataract lenses, and the results are shown in Figure 3. The expressions of filensin were significantly lower in selenite-treated group than they were in normal controls $(p<0.05)$. Moreover, a significant $(p<0.05)$ elevation was observed in the filensin expressions in the groups treated with rosmarinic acid in comparison with those observed in the group treated with selenite. However, the increase was maximum $(p$ $<0.05$ ) in groups of rat pups that received a dose of 50 $\mathrm{mg} / \mathrm{kg}$ rosmarinic acid.
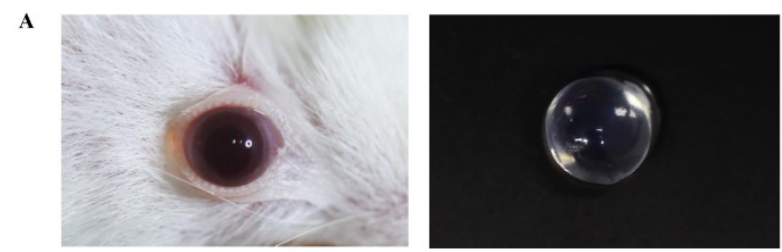

B
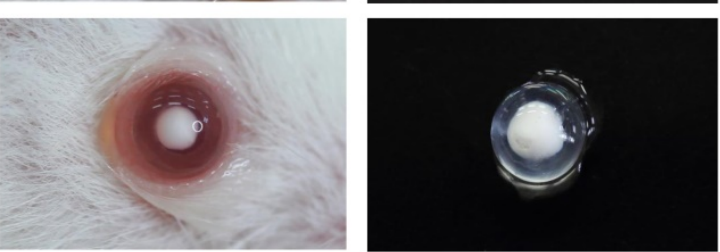

C
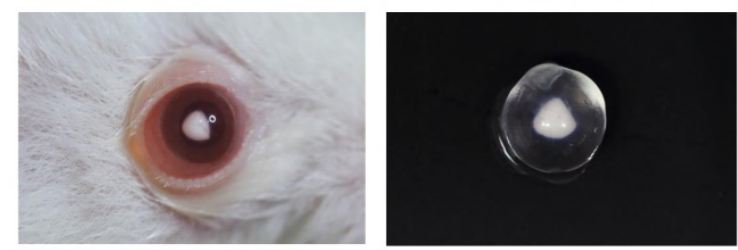

D
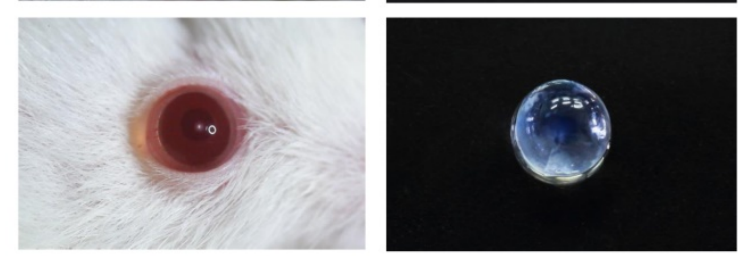

E
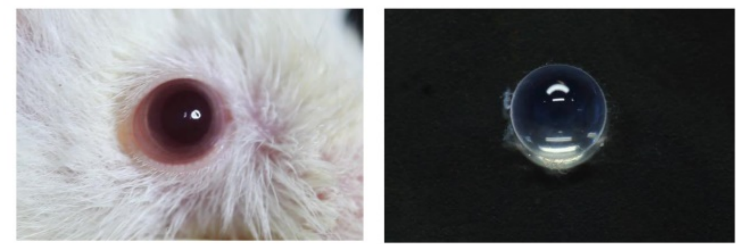

$\mathbf{F}$

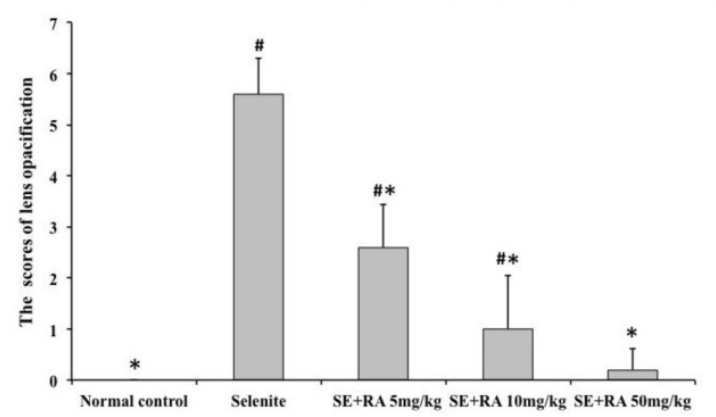

Figure 1. Effect of rosmarinic acid on selenite-induced cataractogenesis. (A) Normal control. (B) Selenite-treated. (C) Selenite +5 $\mathrm{mg} / \mathrm{kg}$ body weight rosmarinic acid. (D) Selenite $+10 \mathrm{mg} / \mathrm{kg}$ body weight rosmarinic acid. (E) Selenite $+50 \mathrm{mg} / \mathrm{kg}$ body weight rosmarinic acid. (F) Effects of rosmarinic acid on the scores of lens opacification in sodium selenite intoxicated rat pups. Values are the mean \pm SD for 10 rat pups; $\# p<0,05$ as compared with normal control. ${ }^{*} p<0.05$ as compared with selenite-treated group. 
A

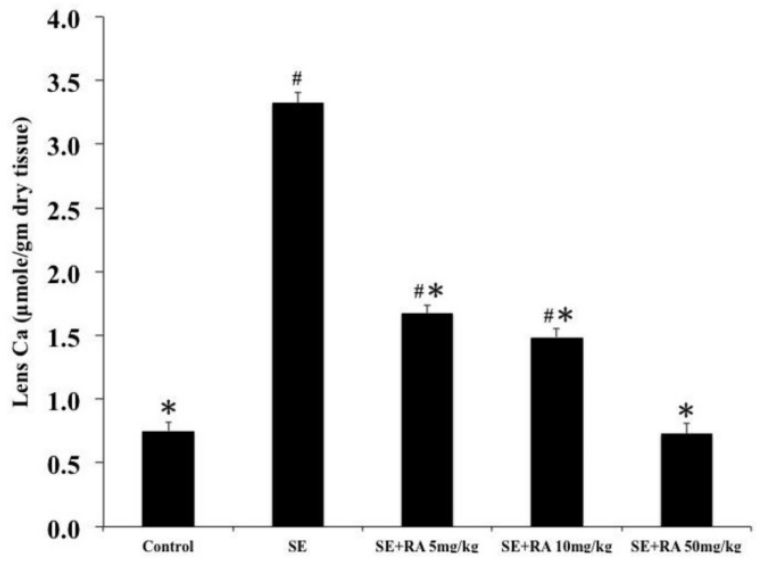

B
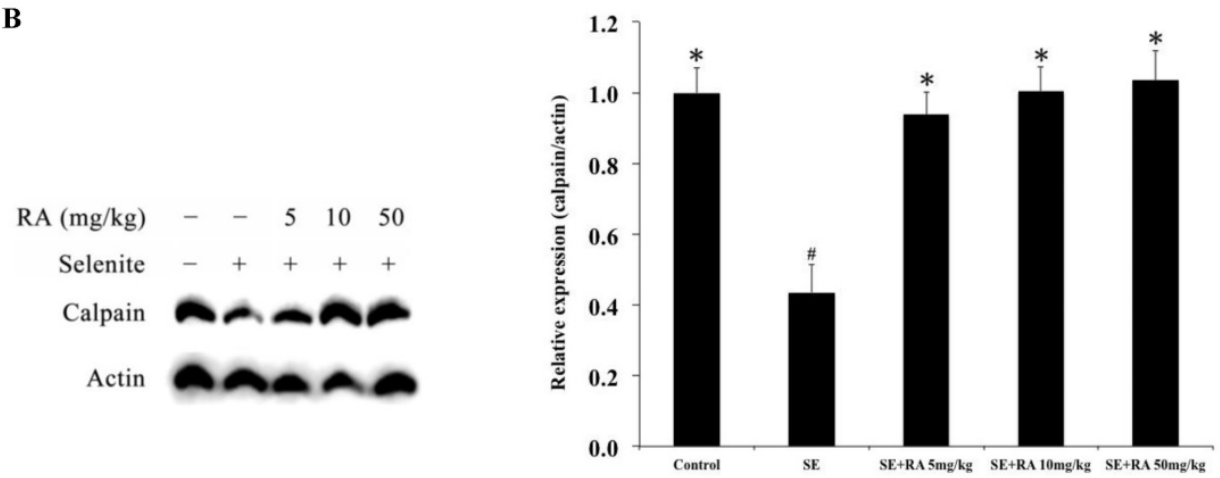

Figure 2. Effect of the rosmarinic acid on lens calcium concentration (A) and calpain expression (B) in selenite-intoxicated rat pups. Western blot analysis to determine the protein levels of calpain and actin was used as the protein loading control. The protein levels of filensin and calpain were quantitatively expressed after being standardized to actin ( $n=3$ at each concentration point). \# $p<0,05$ as compared with normal control. * $p<0.05$ as compared with selenite-treated group.
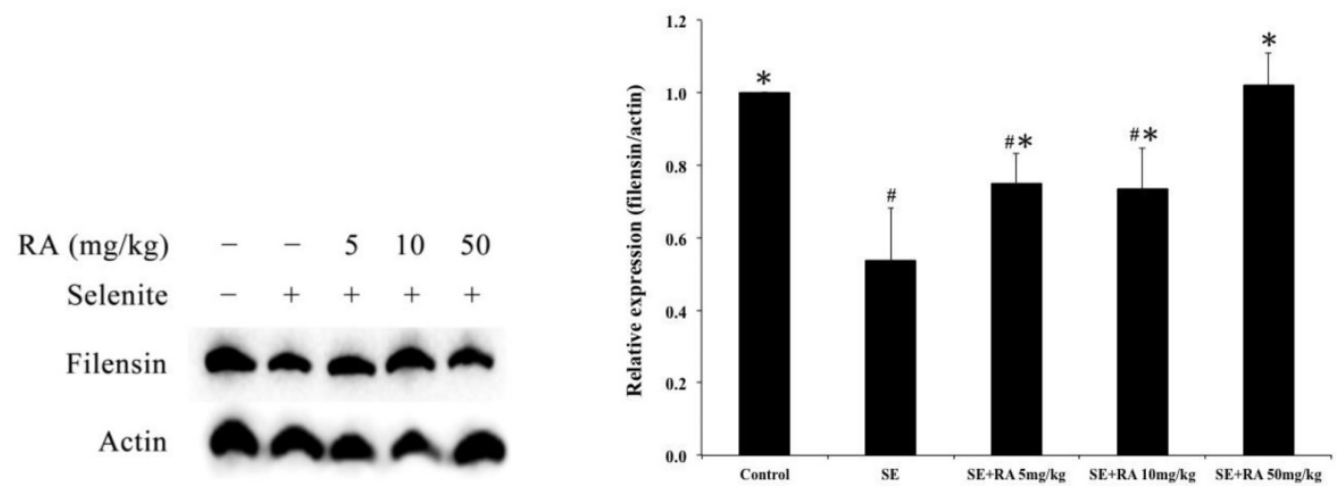

Figure 3. Effect of the rosmarinic acid on lens filensin expression in selenite-intoxicated rat pups. Western blot analysis to determine the protein levels of filensin. Actin was used as the protein loading control. The protein levels of filensin were quantitatively expressed after being standardized to actin ( $n=3$ at each concentration point). $\# p<0,05$ as compared with normal control. ${ }^{*} p<0.05$ as compared with selenite-treated group.

Effects of rosmarinic acid on Nrf2, SOD, HO-1, and NQOI expressions in lenses

The biomarkers of the antioxidant status, such as nuclear factor E2-related factor 2 (Nrf2), superoxide dismutase (SOD), heme oxygenase 1 (HO-1), and the phase II enzyme $\mathrm{NAD}(\mathrm{P}) \mathrm{H}$ : quinone acceptor oxidoreductase 1 (NQO1), were measured to evaluate the oxidation injuries in the lenses. After being normalized and verified with actin, the expressions of Nrf2, SOD, HO-1, and NQO1 were significantly lower $(p<0.05)$ in the lenses that were treated with selenite treatment alone than they were in the lenses of normal control rat pups (Figure 4), suggesting that selenite induced oxidative damage to the lens. However, the rosmarinic acid-treated groups showed significant increases in Nrf2, SOD, HO-1, and NQO1 expression 
at doses of both 10 and $50 \mathrm{mg} / \mathrm{kg}$ compared to the selenite-treated control group $(p<0.05)$. The group treated with rosmarinic acid at a dose of $5 \mathrm{mg} / \mathrm{kg}$ exhibited remarkably amplified $(p<0.05)$ Nrf2 and SOD expressions, whereas the expressions of HO-1 and NQO1 were not significantly affected compared to the selenite-treated control group (Figure 4).

\section{Effect of rosmarinic acid on GSH-PX, GSH-Rd, and catalase activities}

In this study, we measured the activities of the antioxidant enzymes GSH-Px, GSH-Rd, and catalase in the lenses, and the results are shown in Figures 5A, $4 \mathrm{~B}$, and $4 \mathrm{C}$. The activities of GSH-Px, GSH-Rd, and catalase in the lenses of selenite-treated control group were significantly lower $(p<0.05)$ than in the normal control group. Conversely, treatment with rosmarinic acid at the maximum dose of $50 \mathrm{mg} / \mathrm{kg}$ increased the percentages of GSH-Px and GSH-Rd by $22 \%$ and $115 \%$, respectively, compared with the selenitetreated control group (Figures 5A and 5B). Moreover, there was a significant elevation $(p<0.05)$ of catalase, up to $30 \%$ in the group treated with a dose of 50 $\mathrm{mg} / \mathrm{kg}$ rosmarinic acid, but rosmarinic acid treatment at doses of 5 and $10 \mathrm{mg} / \mathrm{kg}$ did not significantly affect the catalase level compared to the selenite-treated control group (Figure 5C).

B
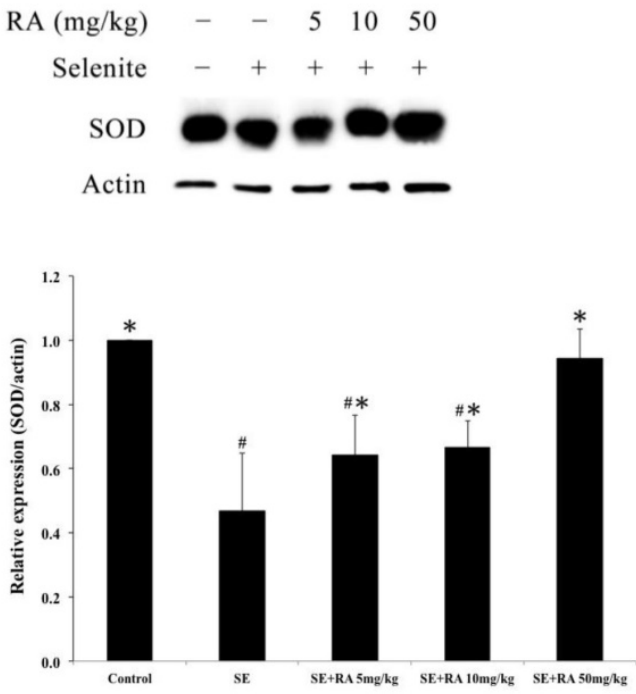

D
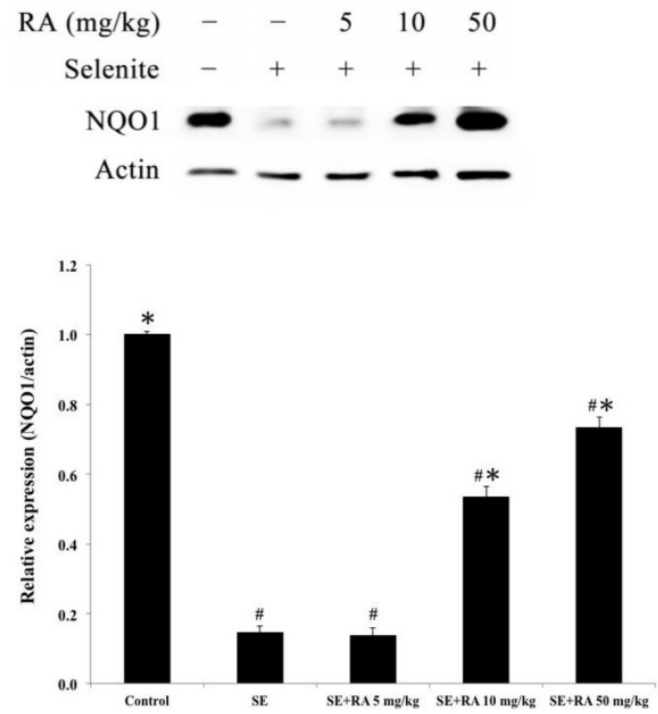

Figure 4. Effect of the rosmarinic acid on lens protein expression in sodium selenite-intoxicated rat pups. Western blot analysis to determine the protein levels of (A) Nrf2, (B) SOD, (C) HO-1 and (D) NQO1. Actin was used as the protein loading control. The protein levels of Nrf2, SOD, HO-1 and NQO1 were quantitatively expressed after being standardized to actin ( $n=3$ at each concentration point). $\# p<0,05$ as compared with normal control. * $p<0.05$ as compared with selenite-treated group. 
A

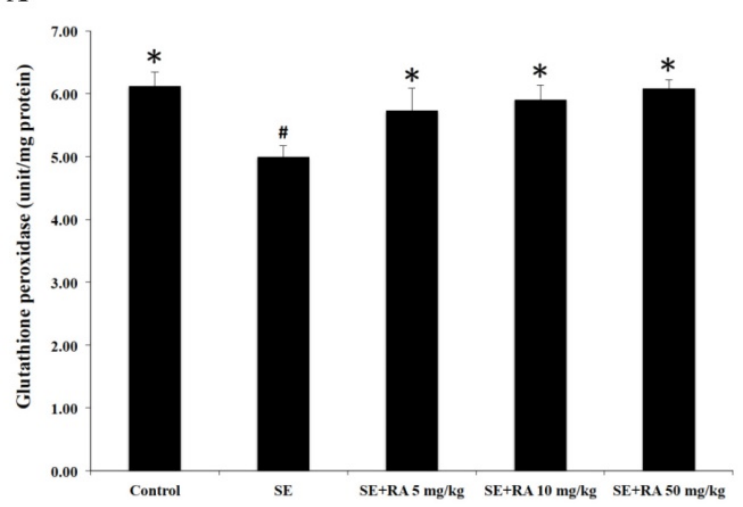

C

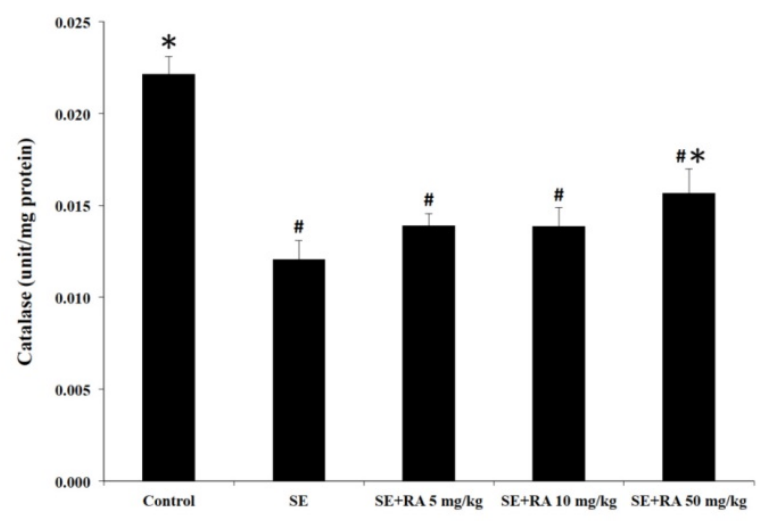

E

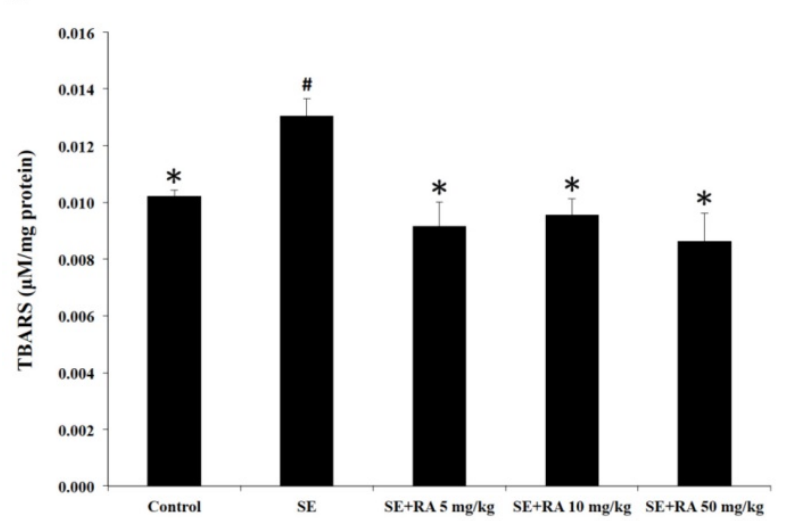

B

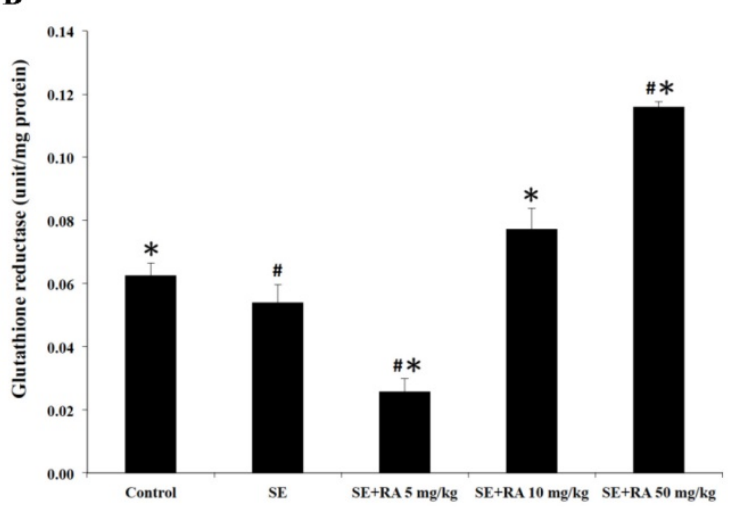

D

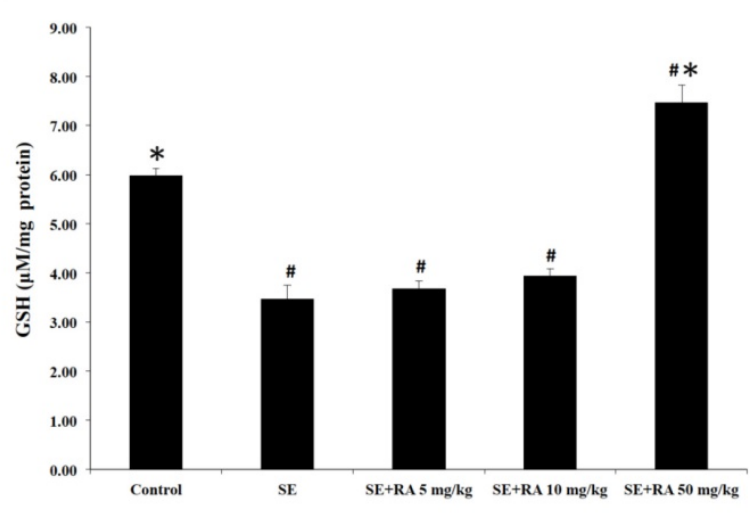

Figure 5. Effects of rosmarinic acid on lens GSH-Px, GSH-Rd, catalase, GSH, and TBARS in sodium selenite intoxicated rat pups. (A) GSH-Px. (B) GSH-Rd. (C) catalase. (D) GSH. (E) TBARS. Values are the mean \pm SD for 10 rat pups; ${ }^{*} p<0.05$ compare with normal control; ${ }^{*} p<0.05$ compare with sodium selenite-treated group.

\section{Effect of rosmarinic acid on GSH and TBARS levels}

GSH is an important nonenzymatic antioxidant in the detoxification pathway that downgrades the reactive toxic metabolites of selenite. The results of the present study demonstrate that selenite caused a significant decrease $(p<0.05)$ in the GSH concentration in the lens when compared to the normal control group. Furthermore, rat pups treated with rosmarinic acid at a dose of $50 \mathrm{mg} / \mathrm{kg}$ showed remarkably increased $(p<0.05)$ GSH concentration, but treatment with rosmarinic acid at doses of 5 and $10 \mathrm{mg} / \mathrm{kg}$ did not significantly affect GSH concentration compared to the selenite-treated control group (Figure 5D).

The TBARS is the general biomarker that appears during the lipid peroxidation of polyunsaturated fatty acids in the biological membrane. The results of the TBARS examinations of the lenses are also shown in Figure 5E. The TBARS concentrations in the selenite-treated group were significantly higher than in the control group $(p<0.05)$. However, the administration of rosmarinic acid significantly 
reduced selenite-induced lipid peroxidation in the lenses. The TBARS concentrations in the rosmarinic acid-treated groups were at least $27 \%$ lower than they were in the selenite-treated control group $(p<0.05)$.

\section{Effects of rosmarinic acid on iNOS, COX-2, and NFKB expressions}

The protein expressions of iNOS, COX-2, and $\mathrm{NFKB}$ are the most frequently used indicators of inflammation. To evaluate the effects of rosmarinic acid on selenite-induced inflammation in the lenses, iNOS, COX-2, and NFKB expressions were determined in this study, and the results are presented in Figure 6. The inflammation-indicating levels of iNOS,
COX-2, and NFKB were significantly higher in the lenses of the selenite-treated group than in the normal control group $(p<0.05)$. However, the groups treated with the maximum dose of rosmarinic acid showed a significantly decreased percentage of selenite-induced iNOS, COX-2, and NFKB expression in the lenses. These protein expressions were reduced by $66 \%, 45 \%$, and $82 \%$, respectively, compared to the selenitetreated group $(p<0.05)$. Similar findings were also found in groups treated with the dose of $10 \mathrm{mg} / \mathrm{kg}$ of rosmarinic acid. These results suggested that the inflammatory protein indicators induced in the lens were effectively inhibited by rosmarinic acid.

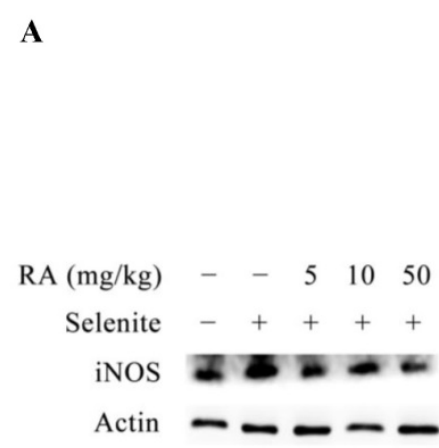

B

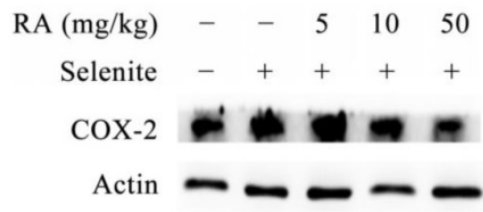

C

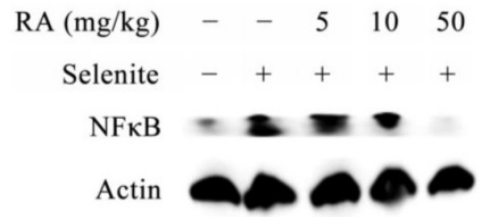

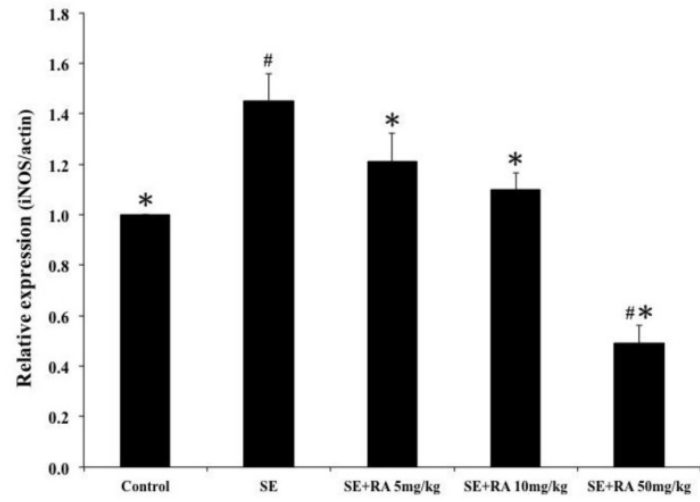
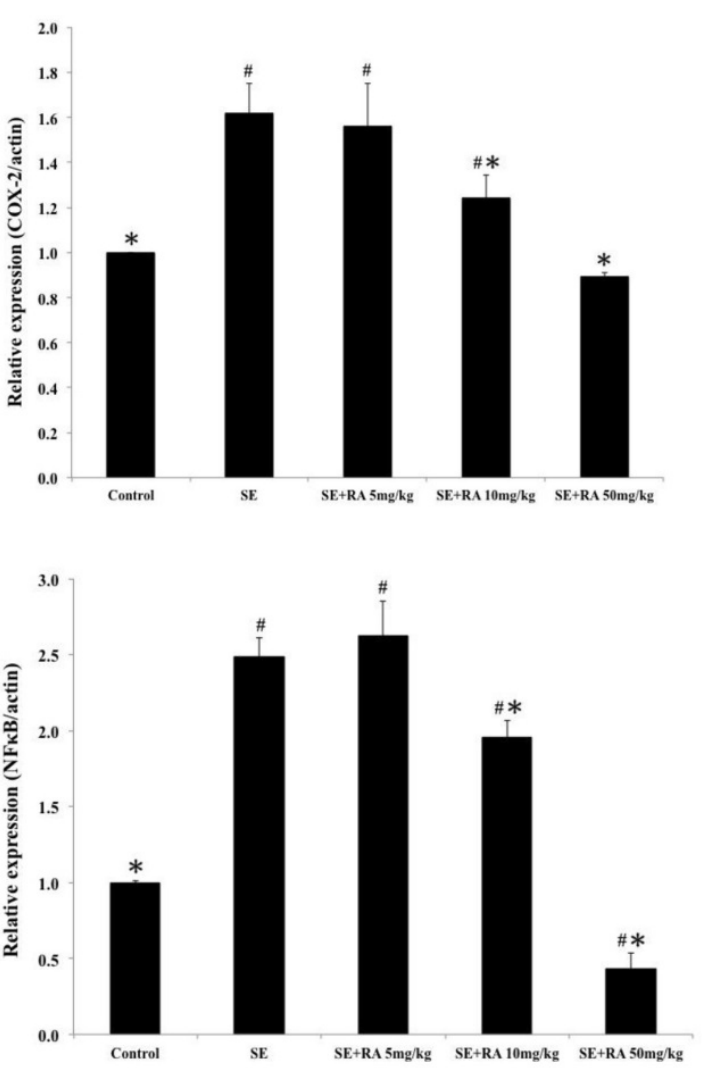

Figure 6. Effect of the rosmarinic acid on lens protein expression in selenite-intoxicated rat pups. Western blot analysis to determine the protein levels of (A) iNOS, (B) COX-2, and (C) NFKB. Actin was used as the protein loading control. The protein levels of iNOS, COX-2, and NFKB were quantitatively expressed after being standardized to actin ( $n=3$ at each concentration point). $\# p<0,05$ as compared with normal control. * $p<0.05$ as compared with selenite-treated group. 
A

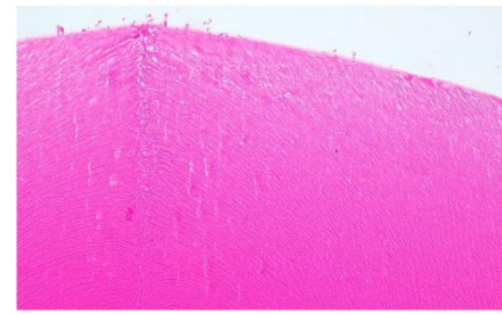

B

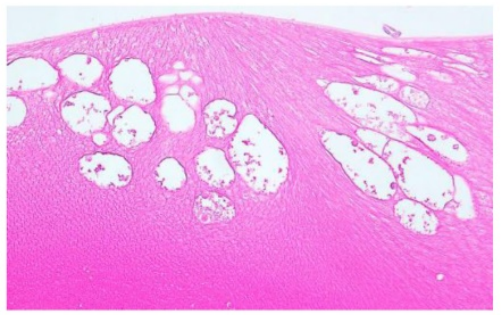

C

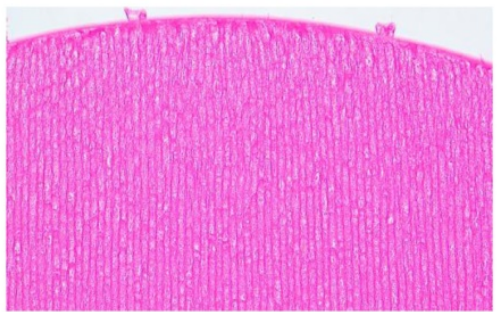

D

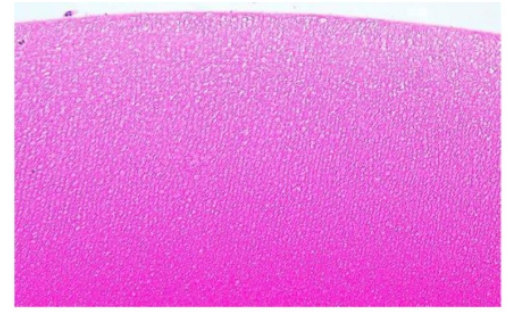

E

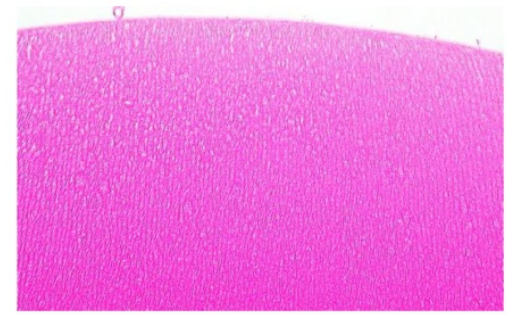

Figure 7. Effect of the rosmarinic acid on lens morphological analysis in selenite-intoxicated rat pups. Lenses were sectioned and stained with hematoxylineosin by standard techniques (200X). (A) Normal control. (B) Selenite-treated. (C) Selenite $+5 \mathrm{mg} / \mathrm{kg}$ body weight rosmarinic acid. (D) Selenite +10 $\mathrm{mg} / \mathrm{kg}$ body weight rosmarinic acid. (E) Selenite $+50 \mathrm{mg} / \mathrm{kg}$ body weight rosmarinic acid.

\section{Histopathologic examination}

The effects of rosmarinic acid in preventing damage to the lenses of the selenite-intoxicated rat pups could be observed by histopathological examination (Fig. 7). In the normal control animals, the histological sections of the lenses displayed normal, tightly packed lens fibers (Figure 7A). The lenses of the selenite-treated rat pups revealed severe injuries, including the lens fibers' degeneration, deformation, swelling, and rupture, with large vacuolization near the lenses' posterior poles (Figure 7B). Compared to the damages observed in the selenite-treated rat pups, the damages of the rosmarinic acid-treated rat pups were much milder or normal. These animals showed only slight swelling of the lens fibers or were as normal as the normal controls (Figures 7C, 7D, and 7E).

\section{Discussion}

An epidemiologic study conducted by the Eye Diseases Prevalence Research Group showed that an estimated 30.1 million Americans will suffer from cataracts in 2020 [19]. Therefore, preventive medical protection as a major defense mechanism against cataracts has been investigated extensively. Overdoses of selenite have often been used for inducing cataracts in rodent pups, and this model shows general similarities to human senile nuclear cataracts, such as the formation of vesicles, increased levels of water-insoluble protein, accumulation of calcium, a decrease in the activity of antioxidant enzymes, and the depletion of GSH [20, 21]. Therefore, we have used sodium selenite-induced cataractogenesis in animal models for investigating the preventive usefulness of rosmarinic acid. Morphological examinations of the lenses treated with sodium selenite showed cataract formation. However, the administration of rosmarinic acid significantly ameliorated and/or delayed cataract formation in the animals' lenses (Figure 1), indicating that rosmarinic acid has the potential to prevent and delay cataracts.

In selenite-induced cataractogenesis models, oxidative stress-induced lipid peroxidative damage of lenticular membranes is a important factor contributing to inhibition of $\mathrm{Ca}^{2+}$-ATPase activity, resulting in the accumulation of calcium in the lens nucleus of selenite cataracts, which, in turn, leads to the activation of lenticular calpains and subsequent proteolytic degradation of lens-soluble proteins. [22, 23]. Accumulation of calcium in the lens nucleus leads to calpain activation, proteolysis, insolubilization, and precipitation of crystallins. We have detected a 4.5-fold increase in calcium levels in the lenses of the selenite-treated group, as compared to the levels in the normal control. Our results are in agreement with other studies that previously reported elevated calcium levels in the lenses of selenite-induced cataracts $[22,24,25]$. However, it is worth noting that treatment of selenite-intoxicated rats with rosmarinic acid prevented such exaltation, and maintained the lens calcium levels close to those of normal control (Fig. 2A).

Calpains, the major calcium-activated proteases in the lenses, have been investigated intensively because their function is highly correlated with lens transparency. In fact, the unregulated activation of calpains directly contributes to cataract formation in animal models. Calpain-mediated degradation of lens crystallin proteins and proteolysis of cytoskeletal elements cause increased light scatter [2]. Several 
studies demonstrated that the administration of sodium selenite could degrade the lenses' intermediate filaments through activation of calpain 2 , leading to the disruption of the cytoskeleton and finally cataract formation [26].

However, decreases in calpain 2 activity appear to occur concomitantly with a rise in lenticular calcium, presumably due to the well-known autolysis of calpain that results from exposure of the enzyme to an elevated calcium concentration [27]. In other words, activation of calpain is followed by degradative autolysis of calpain [28]. Loss of calpain activity after formation of cataract in the mouse model was also postulated to be due to autolysis [29]. Consequently, a decrease in the level of calpain 2 has been reported to occur during selenite cataractogenesis [22]. Contrary to our expectations based on increased calcium levels, decreased calpain 2 levels were observed in the lenses of selenite-treated group. These results could be attributed to the degradative autolysis of calpains 2 subsequent to their activation. Our results are in agreement with other studies that previously reported decreased in both of calpain activity and levels were observed in the selenite-treated group [28]. Their results are also shown that the calpain activity analyzed by casein zymography has consistent results with the calpain expression of Western blot analysis. Furthermore, in the lenses of rosmarinic acid-treated groups, no significant decrease in the level of calpain 2 expression was recorded when compared to the lenses of normal control (Figure 2B) in the present study. Therefore, rosmarinic acid treatment possibly prevented an increase in lenticular calcium levels (Figure 2A), and maintaining lenticular calpain 2 levels at near normal control levels (Figure 2B).

Moreover, filensin is the cell-specific intermediate filaments in the lens and is believed to contribute to the maintenance of lens transparency [30]. In the present study, the rat pups that were treated with sodium selenite alone showed significantly fewer filensin expressions than did the rat pups in the normal control group, implying increased opacity to the lens. On the contrary, we found that treatment with the rosmarinic acid markedly inhibits sodium selenite-induced lenticular opacity as evidenced by elevated filensin expressions in the lenses (Figure 3).

The etiology of cataracts is extremely complicated, but an important mechanism of lens opacity may be related to free radicals that can induce oxidative damage [31,32]. Free radicals are capable of binding to proteins or other molecules, resulting in protein oxidation, a decrease in the capabilities of the antioxidant defense system, structural damage of the crystalline lens, and finally cataract formation [4, 31]. Many studies have shown that a crucial mechanism of protecting the lens against cataract formation may be related to the antioxidant capacities to scavenge reactive oxygen species (ROS) [33,34]. To prevent free radicals from attacking the cells, which would lead to oxidative damage, cells have established an antioxidant defense system to remove the ROS. Therefore, enhancing the intracellular antioxidant defense system and/or inhibiting the production of free radicals are important in protecting the lens from sodium selenite-induced oxidative damage [3]. Nrf2, a major regulator in antioxidant defense, can regulate the expression of antioxidant genes. When motivated by exogenous oxidative stress, Nrf2 translocates to the nucleus from the cytoplasm, thereby binding to antioxidant response elements (AREs). This process also promotes the transcription of antioxidant objectives and finally enhances the intracellular antioxidant defense system. The progression of lens opacification is reportedly associated with the loss of antioxidant enzyme activities. If antioxidant enzyme activities are enhanced, cataractogenesis can be prevented or delayed [35, 36]. The results of the present study report that the expression of Nrf2 in the lens was significantly less in the groups treated with sodium selenite treatment alone than it was in the normal control group. The sodium selenite treatment also appeared to downregulate the levels of SOD, HO-1, NQO1, catalase, GSH-Px, and GSH-Rd in sodium selenite-intoxicated rat pups. By contrast, the rosmarinic acid treatment showed remarkable elevation of the protein expression of $\mathrm{Nrf} 2$ and upregulation of the activities of SOD, HO-1, NQO1, catalase, GSH-Px, and GSH-Rd, implying that rosmarinic acid could restore and/or maintain these enzymes' activities in sodium selenite-damaged lenses (Figure 4 and 5). Similar results from our previous research confirmed that rosmarinic acid prompted the expression of the Nrf2 protein in pterygium epithelial cells and upregulated the activities of enzymes in the antioxidant defense system, such as HO-1, NQO1, SOD, and catalase [13].

Previous studies on the mechanism of sodium selenite-induced oxidative damage in the lens showed that GSH acts as a nonenzymatic antioxidant that reduces ROS, reactive nitrogen species, and xenobiotic compounds [3, 4]. In fact, GSH depletion is significantly correlated with the grade of lens damage [37], suggesting that GSH is essentially necessary for reducing sodium selenite-induced oxidative damage in the lens. Previous studies on the mechanism of sodium selenite-induced oxidative damage in the lens showed that GSH easily reacts with selenite in a non-enzymatic reaction that results in the formation 
of the selenium derivative, GS-Se-SG. Moreover, GSH is easily transformed into glutathione disulfide (GSSG) upon an oxidation reaction with selenite, which may then cause depletion in GSH's intracellular levels. GSSG is either easily decreased by NADPH and glutathione reductase or exploited to assist in the protein-folding processes in the endoplasmic reticulum. Ultimately, GSSG is reduced because it reacts with protein disulfide isomerase to produce GSH. Because of these circulating processes, GSH is a particularly effective intracellular buffer against oxidative stress [38]. Restoring and/or maintaining the GSH levels in the lens may aid in keeping its protective capacity against oxidative stress and eventually postponing the onset of cataracts [39]. In the present study, the lenses' GSH content was significantly less in the sodium selenite-exposed rat pups than it was the control rat pups. However, the group treated with rosmarinic acid had significantly more GSH content in their lenses than did the untreated group (Figure 5D), indicating that rosmarinic acid decreases GSH leakage from the lens.

The TBARS groups are the major indicators that appear during the peroxidation of polyunsaturated fatty acids in biological membranes, and the accumulation of TBARS has been found in numerous human diseases. A rise in TBARS concentrations suggests heightened lipid peroxidation, leading to tissue injury and the antioxidant defense mechanisms' failure to prevent the development of excessive free radicals [40]. Therefore, TBARS concentrations are essentially the most typical biomarkers of lipid peroxidation. In the present study, the TBARS contents of the lenses significantly increased in the sodium selenite-treated group; however, the rosmarinic acid-treated group had significantly decreased TBARS levels (Figure 5E).

The etiology of cataracts includes oxidative stress, inflammation, and apoptotic factors, which, because of rapid increases in reactive oxygen or nitrogen species and lipid peroxidation, lead to the breakdown of proteins and finally to the opacification of the lens. However, the NFKB pathway is one of the major pathways of cellular stress and inflammation. The transcription factor NF- $\mathrm{KB}$ is a crucial mediator of the immune response, regulating the expression of pro-inflammatory enzymes and cytokine receptors, which are involved in inflammation, cell proliferation, and apoptosis [41]. Upon stimulation with oxidative stress, NF-KB is released from an inhibitory subunit (ІкB) and allowed to translocate the factor's heterodimer into the nucleus, where it promotes the transcriptional activation of several pro-inflammatory enzymes that include iNOS and COX-2 [42]. Moreover, nitric oxide derived from iNOS and prostaglandin E2, which is synthesized by COX-2, plays a central role in the pathogenesis of acute and chronic inflammation. A previous study indicated that rosmarinic acid inhibits TPA-induced inflammatory responses by inhibiting the NF-kB pathway [43]. In the present study, the expression of proinflammatory cytokines, including NFKB, iNOS, and COX-2 were increased by a sodium selenite treatment. However, rosmarinic acid showed potent antiinflammatory activity, including the down-regulation of $\mathrm{NFKB}$ activation and consequent suppressions of iNOS and COX-2 expression (Figure 6).

Based on our findings, this study provides evidence of the preventive effect of rosmarinic acid on sodium selenite-induced cataracts in the rat pups. Our results show that the protective effects of rosmarinic acid come from its inhibiting the processes of lipid peroxidation and inflammation, as well as the acid's increase of the activity of the antioxidant defense system. In the final analysis, our results suggest that rosmarinic acid can ameliorate sodium seleniteinduced cataractogenesis in rat pups.

\section{Competing Interests}

The authors have declared that no competing interest exists.

\section{References}

1. Resnikoff S, Pascolini D, Etya'ale D, et al. Global data on visual impairment in the year 2002. Bull World Health Organ. 2004; 82(11): 844-51.

2. Rooban BN, Lija Y, Biju PG, et al. Vitex negundo attenuates calpain activation and cataractogenesis in selenite models. Exp Eye Res. 2009; 88(3): 575-82.

3. Shearer TR, Ma H, Fukiage C, et al. Selenite nuclear cataract: review of the model. Mol Vis. 1997; 3:8.

4. Ho MC, Peng YJ, Chen SJ, et al. Senile cataracts and oxidative stress. Journal of Clinical Gerontology and Geriatrics. 2010; 1(1): 17-21.

5. Manikandan R, Thiagarajan R, Beulaja S, et al. Effect of curcumin on selenite-induced cataractogenesis in Wistar rat pups. Curr Eye Res. 2010; 35(2): $122-9$

6. Doganay S, Borazan M, Iraz M, et al. The effect of resveratrol in experimental cataract model formed by sodium selenite. Curr Eye Res. 2006; 31(2): 147-53.

7. Petersen M, Simmonds MS. Rosmarinic acid. Phytochemistry. 2003; 62: 121-5.

8. Petersen M, Abdullah Y, Benner J, et al. Evolution of rosmarinic acid biosynthesis. Phytochemistry. 2009; 70: 1663-79.

9. Sanchez-Campillo M, Gabaldon JA, Castillo J, et al. Rosmarinic acid, a photo-protective agent against $\mathrm{UV}$ and other ionizing radiations. Food Chem Toxicol. 2009; 47: 386-92.

10. Vostalova J, Zdarilova A, Svobodova A. Prunella vulgaris extract and rosmarinic acid prevent UVB-induced DNA damage and oxidative stress in HaCaT keratinocytes. Arch Dermatol Res. 2010; 302: 171-81.

11. Zhang JJ, Wang YL, Feng XB, et al. Rosmarinic acid inhibits proliferation and induces apoptosis of hepatic stellate cells. Biol Pharm Bull. 2011; 34: 343-8.

12. Wu CF, Hong C, Klauck SM, et al. Molecular mechanisms of rosmarinic acid from Salvia miltiorrhiza in acute lymphoblastic leukemia cells. J Ethnopharmacol. 2015; 176: 55-68.

13. Chen YY, Tsai CF, Tsai MC, et al. Inhibitory effects of rosmarinic acid on pterygium epithelial cells through redox imbalance and induction of extrinsic and intrinsic apoptosis. Exp Eye Res. 2017; 160: 96-105.

14. Chemerovski-Glikman $\mathrm{M}$, Mimouni M, Dagan $\mathrm{Y}$, et al. Rosmarinic Acid Restores Complete Transparency of Sonicated Human Cataract Ex Vivo and Delays Cataract Formation In Vivo. Sci Rep. 2018; 8(1): 9341.

15. al-Sereiti MR, Abu-Amer KM, Sen P. Pharmacology of rosemary (Rosmarinus officinalis Linn.) and its therapeutic potentials. Indian J Exp Biol. 1999; 37(2): 124-30.

16. Velpandian T, Gupta P, Ravi AK, et al. Evaluation of pharmacological activities and assessment of intraocular penetration of an ayurvedic polyherbal eye drop (Itone ${ }^{\mathrm{TM}}$ ) in experimental models. BMC Complement Altern Med. 2013; 13: 1.

17. Khan S, Bhardwaj T, Somvanshi P, et al. Inhibition of C298S mutant of human aldose reductase for antidiabetic applications: Evidence from in silico 
elementary mode analysis of biological network model. J Cell Biochem. 2018; 119(8): 6961-73.

18. Hiraoka T, Clark JI, Li XY, et al. Effect of selected anti-cataract agents on opacification in the selenite cataract model. Exp Eye Res. 1996; 62: 11-9.

19. Congdon N, Vingerling JR, Klein BE, et al. Prevalence of cataract and pseudophakia/aphakia among adults in the United States. Arch Ophthalmol. 2004; 122(4): 487-94.

20. Orhan H, Marol S, Hepsen IF, et al. Effects of some probable antioxidants on selenite-induced cataract formation and oxidative stress-related parameters in rats. Toxicology. 1999; 139: 219-32.

21. Qi HP, Wei SQ, Gao XC, et al. Ursodeoxycholic acid prevents selenite-induced oxidative stress and alleviates cataract formation: In vitro and in vivo studies. Mol Vis. 2012; 18: 151-60.

22. Hightower KR, David LL, Shearer TR. Regional distribution of free calcium in selenite cataract: relation to calpain II. Invest Ophthalmol Vis Sci. 1987; 28(10): $1702-6$.

23. Wang $\mathrm{Z}$, Bunce GE, Hess JL. Selenite and $\mathrm{Ca}^{2+}$ homeostasis in the rat lens: effect on $\mathrm{Ca}^{2+}-\mathrm{ATPase}$ and passive $\mathrm{Ca}^{2+}$ transport. Curr Eye Res. 1993; 12(3): 213-8

24. Elanchezhian R, Sakthivel M, Geraldine P, et al. The effect of acetyl-L-carnitine on lenticular calpain activity in prevention of seleniteinduced cataractogenesis. Exp Eye Res. 2009; 88(5): 938-44.

25. Robertson LJ, Morton JD, Yamaguchi M, et al. Calpain may contribute to hereditary cataract formation in sheep. Invest Ophthalmol Vis Sci. 2005; 46(12): 4634-40.

26. Mathur P, Gupta SK, Wegener AR, et al. Comparison of various calpain inhibitors in reduction of light scattering, protein precipitation and nuclear cataract in vitro. Curr Eye Res. 2000; 21(6): 926-33.

27. Suzuki, K. Calcium activated neutral protease: domain, structure and activity regulation. Trends Biol Sci. 1987; 12: 103

28. Yoshida $\mathrm{H}$, Murachi T, Tsukahara I. Age-related changes in calpain II and a-crystallin in the lens of hereditary cataract (Nakano) mouse. Curr Eye Res. 1985; 4: 983

29. Maddirala $\mathrm{Y}$, Tobwala $\mathrm{S}$, Karacal $\mathrm{H}$, et al. Prevention and reversal of selenite-induced cataracts by $\mathrm{N}$-acetylcysteine amide in Wistar rats. MBC Ophthalmol. 2017; 17: 54-65.

30. Oka M, Kudo H, Sugama N, et al. The function of filensin and phakinin in lens transparency. Mol Vis. 2008; 14: 815-22.

31. Spector A. Oxidative stress-induced cataract: mechanism of action. FASEB. 1995; 9(12): 1173-82.

32. Biju PG, Rooban BN, Lija Y, et al. Drevogenin D prevents selenite-induced oxidative stress and calpain activation in cultured rat lens. Mol Vis. 2007; 13: $1121-9$

33. Thiagarajan R, Manikandan R. Antioxidants and cataract. Free Radic Res. 2013; 47(5): 337-45

34. Kumari RP, Ramkumar S, Thankappan B, et al. Transcriptional regulation of crystallin, redox, and apoptotic genes by C-Phycocyanin in the selenite-induced cataractogenic rat model. Mol Vis. 2015; 21: 26-39.

35. Shinohara $\mathrm{T}$, White $\mathrm{H}$, Mulhern ML, et al. Cataract: window for systemic disorders. Med Hypotheses. 2007; 69: 669-77.

36. Shichi H. Cataract formation and prevention. Expert Opin Investig Drugs. 2004; 13: 691-701.

37. Nakazawa $\mathrm{Y}, \mathrm{Oka} \mathrm{M}$, Bando $\mathrm{M}$, et al. Hesperetin prevents selenite-induced cataract in rats. Mol Vis. 2015; 21: 804-10.

38. Cantin $\mathrm{AM}$, White $\mathrm{TB}$, Cross $\mathrm{CE}$, et al Antioxidants in cystic fibrosis Conclusions from the CF Antioxidant Workshop, Bethesda, Maryland, November 11-12, 2003. J Free Radic Med. 2007; 42: 15-31.

39. Harding JJ. Can drugs or micronutrients prevent cataract? Drugs Aging. 2001; 18: 473-86.

40. Vaca CE, Wilhelm J, Harms-Rihsdahl M. Interaction of lipid peroxidation product with DNA. A review. Mutation Res. 1988; 195: 137-49.

41. Martindale JL, Holbrook NJ. Cellular response to oxidative stress: signaling for suicide and survival. J Cell Physiol. 2002; 192: 1-15.

42. Terquankar V. NFkappa B pathway: a good signaling paradigm and therapeutic target. Int J Biochem Cell Biol. 2006; 38: 1647-53.

43. Osakabe N, Yasuda A, Natsume M, et al. Rosmarinic acid inhibits epidermal inflammatory responses: anticarcinogenic effect of Perilla frutescens extract in the murine two-stage skin model. Carcinogenesis. 2004; 25(4): 549-57. 